\title{
Filigrane
}

Écoutes psychothérapiques

\section{Les fondements d'une parole vraie en psychanalyse. Un entretien avec Guy Da Silva}

\section{Réal Laperrière}

Volume 19, numéro 2, automne 2010

URI : https://id.erudit.org/iderudit/1000461ar

DOI : https://doi.org/10.7202/1000461ar

Aller au sommaire du numéro

Éditeur(s)

Revue Santé mentale au Québec

ISSN

1192-1412 (imprimé)

1911-4656 (numérique)

Découvrir la revue

Citer ce document

Laperrière, R. (2010). Les fondements d'une parole vraie en psychanalyse.

Un entretien avec Guy Da Silva. Filigrane, 19(2), 95-113.

https://doi.org/10.7202/1000461ar d'utilisation que vous pouvez consulter en ligne. 


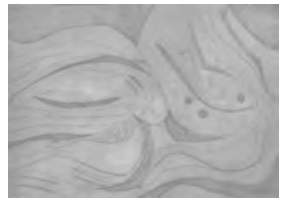

\title{
Les fondements d'une parole vraie en psychanalyse. Un entretien avec Guy Da Silva
}

\author{
Réal Laperrière
}

\begin{abstract}
M embre de la Société psychanalytique de Montréal, Guy Da Silva pratique la psychanalyse depuis 37 ans. D'abord intéressé par les lettres et par le théâtre - il a été membre des Comédiens routiers du Gésu puis des Compagnons de Saint-Laurent, il étudie la médecine à l'Université de Montréal dans les années cinquante puis part faire sa spécialisation en psychiatrie à Boston, où il réside pendant cinq ans et y entreprend une formation psychanalytique qui sera complétée plus tard à Montréal, au Québec English (Section anglophone de la Société canadienne de psychanalyse). C'est dans un hôpital psychiatrique de Boston qu'il s'intéresse à la schizophrénie, à la psychodynamique de la personne âgée ainsi qu'à l'approche psychanalytique des groupes, intérêt qu'il développera tout au long de sa carrière. À son retour au Québec en 1962, il travaille à l'Institut Albert-Prévost, où il a été directeur de la Clinique Externe pendant dix ans, et y enseigne et pratique jusqu'en 2003. Au cours de ces années, il exerce parallèlement en privé et comme consultant dans de nombreuses institutions montréalaises (Jewish General Hospital, Allan Memorial Institute, Hôpital Notre-Dame, Centre d'Orientation, Sir Georges William College, Centre de réadaptation La Clairière). Professeur agrégé de clinique à l'Université de Montréal de 1994 à 2003, membre didacticien de l'Institut canadien de psychanalyse, il a contribué à la formation de très nombreux psychiatres, psychanalystes et psychothérapeutes québécois, francophones et anglophones. L'entretien a eu lieu à son cabinet de la rue Pratt, à Outremont.
\end{abstract}

R. L. : Si vous le voulez bien Docteur Da Silva, nous allons débuter cet entretien en abordant votre article paru dans l'International Journal of Psychoanalysis, lequel consistait en l'élaboration d'un texte que vous aviez présenté auparavant à la Société psychanalytique de Montréal.

G. D. S.: Je l'avais présenté en 1984. On m'avait dit: "C'est intéressant. C'est spécial.» Alors je l'ai retravaillé avec un bon nombre d'exemples cliniques, et présenté à nouveau pour l'élection comme didacticien en 1987. 
R. L. : Vous vous étiez basé sur deux idées, l'une de Freud sur l'expérience de satisfaction et l'autre de Bion, en rapport avec l'appareil à penser, construit en analogie avec le modèle de l'appareil digestif.

G. D.S. : Exactement. Vous avez raison. Freud et l'expérience de satisfaction, et Bion, le modèle digestif pour la construction de l'appareil à penser. Ces observations-là venaient de ma propre analyse à moi, en tant qu'analysé, mais aussi chez mon analyste, ça m'avait frappé, ces bruits de ventre venant de lui mais aussi de moi.

L'idée, en fait, qui m'est apparue c'était que les borborygmes étaient des indicateurs du travail psychique en cours. Bion n'avait pas parlé des borborygmes comme tels. J'avais discuté de ça avec Clifford Scott à l'époque qui m'avait encouragé: «Essayez de tenir compte aussi des autres sons du corps. » Parce qu'il était très intéressé à cela quand j'en avais discuté avec lui. Il disait : «Par exemple, les rots ou les flatus etc.» Mais bon, j'ai voulu me concentrer sur les borborygmes. Il y avait eu certaines allusions dans la littérature par Freud, mais sans l'élaborer et puis par Scott aussi. Mais l'idée fondamentale ou une des idées fondamentales, m'était venue d'une citation d'André Green...

R. L.: Green?

G. D. S. : Oui, quand il est venu, à Montréal... mais ça, c'est plus tard. En revoyant mes notes, j'ai pensé à la présentation de Green en 1996 sur l'intrapsychique et l'intersubjectif en psychanalyse. Et une phrase en particulier qu'il avait citée, de Freud, alors que Freud faisait allusion au trouble de la pensée. Cette phrase de 1932 était la base de la conférence de Green : «Sur le chemin de la pulsion vers l'objet, la pulsion se psychise progressivement. » C'était la première fois que Freud parlait de ça, qu'il y avait une transformation de quelque chose de pulsionnel qui devenait psychisé. Cette transformation pour moi, ça c'était fondamental! Et j'ai pensé donc que «pour une parole vraie, les trois sens du mot sens doivent être réunis: la sensation, le sentiment, c'est-à-dire l'émotion, et le sens des mots. » Ça, c'est vraiment fondamental. Alors, qu'est-ce qui est primaire? Au fond, la question était posée : est-ce que c'est la satisfaction du besoin instinctuel ou est-ce que c'est la part de la relation avec un objet? Et quel est le lien entre les deux, besoin instinctuel et besoin relationnel?

Il y a des expressions linguistiques qu'on peut retrouver dans la littérature; j'ai fouillé cela au moment d'écrire le texte. Par exemple, le latin degerere a donné le mot digest. Au dictionnaire "degerere », c'est mûrir par la réflexion. Aussi, en latin, le mot pensare qui est en français " penser", en anglais pansy, qui vient aussi du latin pentices: guts en anglais. Autrement dit, il y a dans le vocabulaire, dans la langue, dans la linguistique si on veut, des liens qui sont faits, entre l'idée de penser et quelque chose qui vient de la digestion avant même que ce soit présenté et élaboré par Bion. Mais dans l'œuvre de Bion, je n'ai pas vu qu'il parlait des borborygmes en tant que signal de cela.

Pour moi donc, l'appareil à penser serait calqué sur le modèle digestif. Prenons pour exemple la question de la mémoire. Pour Freud, la mémoire vient par la pensée: c'est un souvenir qui a été pensé. Pour Klein, je me souviens très bien que c'est elle qui, pour la première fois, a introduit dans un texte sur l'identification projective: memories in feelings. Plutôt que la mémoire soit liée seulement à la pensée, elle 
introduit, mais sans l'élaborer, l'idée que les sentiments, les affects peuvent aussi, en fait, rappeler quelque chose. Memories in feeling, c'était son thème. Mais elle n'a pas élaboré plus là-dessus. C'est vraiment Bion qui lui, a parlé de memories in sensations. Si l'on prend les trois auteurs, Freud, Klein et Bion, on voit la progression au point de vue de la mémoire. Pour Freud c'est par la pensée, pour Klein c'est aussi par l'affect, et c'est Bion qui introduit en plus la mémoire à partir des sensations dans le corps: memories in bodily sensations.

R. L.: Donc, pour vous, les borborygmes pendant la séance seraient des marqueurs du travail de quelque chose qui serait à digérer mentalement?

G. D.S. : C'est ça, c'est là-dessus que le texte est centré. C'est-à-dire que si on entend des bruits de ventre à certains moments, c'est en fait qu'il s'agit d'un travail intérieur de transformation. Tout le texte était basé là-dessus. Une nouvelle idée ou la difficulté d'accepter une nouvelle idée va être élaborée à la fois par la pensée, et en même temps ce sera accompagné par des bruits, par des borborygmes signalant en quelque sorte une « digestion mentale» de cette nouvelle idée. J'ai donné dans le texte des exemples cliniques.

R. L.: Vous allez assez loin, parce que vous identifiez même des tonalités différentes dans le son des borborygmes. C'est un peu comme si vous proposiez d'écouter la musique de la séance, et pas seulement le contenu verbal, pas seulement le discours. G. D. S.: C'est l'ensemble. À quel moment du discours (les mots) et de l'émotion accompagnant ce discours, ces bruits-là vont apparaître. Bion est le premier analyste à reconnaître l'importance de l'expérience émotionnelle au-delà du discours. Fondamentalement, sa théorie est basée là-dessus; c'est l'épicentre de sa théorie. Auparavant, avec Freud, l'émotion était simplement un facteur quantitatif. Tandis que pour Bion, l'expérience émotionnelle, c'est ce qui donnait le plus de sens, qui allait faire surgir le sens.

Si je relis mes notes:

— construction d'un appareil basé sur le système digestif, par l'observation du système alimentaire

- les borborygmes en tant que marqueur du travail psychique en séance; le marqueur de la digestion, de l'expérience émotionnelle en cours, à ce moment-là de l'analyse.

J'avais fait déjà allusion au cas de Zénon, dans le texte de 1987, où il était question d'une dépression existentielle, d'un individu stoïque, sans émotion, qui n'avait jamais, lui, éprouvé de vraie dépendance infantile. Et c'est en fait au moment où il commençait pour la première fois de sa vie, en analyse, à éprouver la dépendance on the analytic breast. Il y a du Klein là-dedans. Et puis, j'avais aussi mentionné le cas de Monsieur Miserabilis, un homme qui avait des idées suicidaires et dont la mère était en dépression post-partum à sa naissance. À un moment donné, en l'écoutant, il m'était venu, en séance, un chant plaintif de mon enfance. J'ai commencé à le chantonner moi-même et à ce moment-là, le patient se rappelait exactement cette fameuse chanson qui était bien connue à l'époque: Ego sum pauper, nihil habeo et nihil dabo... Vous connaissez? R. L.: Non. 
G. D. S. : Ce qui veut dire: «Je suis pauvre, je n'ai rien, et je ne donnerai rien.» Il avait été question de sa difficulté d'arriver à changer. Et puis, à un moment donné, il avait commencé à manger des papayas. Je ne connaissais pas ça, moi. En association, tout à coup, ce qu'il pensait c'était: «Papa est là.» Et le papa, c'était l'analyste. Il y a un papa qui était là, pour lui, à ce moment-là.

En fait, le travail de pensée commence par l'observation des sensations corporelles et par après, ça se poursuit vers de plus en plus d'abstraction. Mais ça, c'est la grille élaborée par Bion. Et moi à ce moment de l'analyse: «I had become in the transference, a thinking mother, feeding his computing brain. A learning from emotional experience was taking place. » Ce sont les notes que j'avais écrites dans mon texte de 1987.

R. L. : J'aimerais maintenant que l'on s'attarde un peu à votre texte de $1994 \ldots$

G. D.S. : «Deuil et mélancolie»?

R. L.: Voilà. Il faut dire que c'est un travail qui a été présenté à un "Congrès International sur les processus de guérison » et j'ai trouvé que vous aviez écrit de très belles pages sur le deuil, la dépression et j'ai retenu certaines choses...

G. D. S. : Ce texte-là, n'a pas été publié mais je souhaiterais qu'il le soit.

R. L.: Oui, moi, je trouvais qu'il y avait des choses importantes là-dedans. Entre autres, vous parlez du travail psychique indispensable pour arriver à sortir le mort du vivant, surtout dans la dépression mélancolique, j’imagine. Et vous utilisez trois termes latins : morbus, morsus et mortuus. Et pour résumer votre position : la morbidité de la maladie (morbus) vient de la morsure (morsus) du mort (mortuus).

G. D. S. :... à l'intérieur de l'individu.

R. L.: C'est ça. Autrement dit, vous dites : ce qui fait la maladie, c'est que l'objet idéalisé, mort ou perdu à l'extérieur de soi, est maintenant incorporé à l'intérieur de soi par un mouvement régressif d'attaque cannibalique de dévoration. Et cet objet mort ou agonisant est maintenu ainsi dans un état endommagé et persécuteur.

G. D. S. : Ces idées que j'ai mises dans ce texte-là, fondamentalement, elles viennent d'Henri Rey. Je suis allé à l'île de Ré plusieurs fois où il avait sa maison pour le rencontrer. Ça a été vraiment une rencontre qui m’a beaucoup, beaucoup apporté. J'ai été très touché par ce monsieur-là. Je l'ai rencontré plusieurs fois, par après, quand il a cessé de voyager et d'enseigner de par le monde.

R. L. : Alors, c'est lui qui vous a inspiré cette idée-là

G. D. S.: Oui. J'avais présenté mon texte à un congrès international sur le processus de guérison, en 1994, mais par après je l'ai présenté un peu partout, y compris à Albert-Prévost en 1994. Ce qui avait déchaîné des passions auprès des psychiatres qui commençaient, à ce moment-là, à être emballés par les nouvelles molécules qui, disaient-ils, allaient sonner le glas de la psychanalyse. Il y a eu un mouvement antipsychanalytique assez important à un moment donné. Alors qu'au début, quand je suis arrivé là, à « Prévost » on n'en avait que pour la psychanalyse avec Camille Laurin. Par la suite, j'ai présenté ce texte aux analystes en formation à la SPM et à l'IPM, un texte grandement inspiré par mon professeur bien-aimé, $D^{r}$ Henri Rey.

Comment sortir le mort du vivant? Il insistait beaucoup là-dessus : le deuil n'est pas une maladie, il y a le travail de deuil versus la mélancolie. Dans la mélancolie, le 
mort est toujours dans le vivant. Dans le deuil normal, il y a une réalité, et il y a du mouvement. Dans l'expression, par exemple, Vita est in motu, la vie est dans le mouvement. Tandis que dans la mélancolie, l'objet est à la fois idéalisé et persécuteur. Ça va toujours ensemble. Quand on idéalise quelqu'un, ça se retourne contre soi, d'une certaine manière. On s'abandonne pour l'autre, finalement. Il y a, dans la mélancolie, une régression. Il faut, et Henri Rey a insisté beaucoup là-dessus, il faut, à ce moment-là, remonter à la période de la vie où le symptôme physique est apparu.

R. L.: Le symptôme physique?

G. D. S. : Le symptôme physique, par exemple, la constipation, le retard mental ou même l'arrêt de développement... l'importance de remonter au début, à l'apparition du symptôme.

Il y a des distinctions qui sont faites dans ce texte entre, d'une part, la dépression réactionnelle et d'autre part, la dépression endogène. Henri Rey donnait l'exemple de la jument versus le singe. Si une jument perd son poulain, elle va aller à la rivière, elle va rester là et elle va s'en aller, c'est tout. En contraste, le singe qui perd un bébé a des sentiments, des pleurs, des cris, etc. Donc, il y a vraiment une période de manifestations d'endeuillé comme chez les humains.

Et Henry Rey notait la différence entre la tristesse et la dépression. Dans la dépression il y a de la culpabilité: on se sent coupable de la mort de l'objet. Et il faut absolument interpréter le sentiment d'omnipotence et aussi la culpabilité par rapport à la perte, les reproches qu'on se fait à soi et les reproches qu'on fait aussi à l'objet perdu. Il y a, on l'a mentionné tantôt, la présence de mort dans le vivant à ce moment-là. Un bon exemple est le roman L'Arrache-cœur.

R. L.: De Boris Vian.

G. D. S. : Oui, dans lequel le psychiatre est chargé d'être le vidangeur de la rivière qui passe dans le village, en mangeant les cadavres qui circulent dans cette rivière. J'ai donné un exemple d'une patiente pour laquelle j'ai été, moi, le vidangeur. C'était comme ça, elle apportait ses déchets en entrevue d'une certaine manière.

La deuxième partie du texte était à propos de la relation du travail psychique et de la médication.

R. L.: Oui, c'est important ça...

G. D. S. : Et c'est à ce propos que ma présentation du texte a été très critiquée par les gens à Prévost; à cette époque commençait l'intérêt pour les nouvelles molécules. Il y a eu une période à Prévost - là, ça c'est un peu atténué mais pas complètement où il y avait beaucoup d'hostilité par rapport à la psychanalyse, avec l'arrivée des nouvelles molécules.

Alors, quelques citations, ici.

Bion, par exemple: «L'être humain est confronté à la nécessité d'avoir à penser pour survivre, et à la terreur d'avoir à penser.»

Et puis une citation de Freud dans ce texte: «La vie est lourde, peu de gens peuvent se passer d'une forme quelconque de drogue » pour tolérer la vie.

«Le risque de suicide, c'est quand le patient n'a plus espoir de réparer l'objet interne endommagé.» Ça, c'est du André Green. 
L'important pour le thérapeute, c'est de tolérer sa propre impuissance face à sa propre mortalité pour pouvoir aider les patients. Deuxièmement, toujours du côté du thérapeute, de tolérer son envie face aux êtres chers qui vont lui survivre. Et troisièmement, la nécessité de tolérer sa propre solitude existentielle.

R. L.: Dans ce texte, vous faites un plaidoyer pour un certain degré de souffrance mentale. C'est-à-dire que pour vous, la souffrance mentale, que ce soit la dépressivité ou l'anxiété, n'est pas un symptôme à faire disparaître par la médication automatiquement. Pour vous, c'est un symptôme qui alerte l'individu sur la nécessité de réexaminer certains aspects de sa vie. Vous le soulevez dans le texte. C'est un texte qui date de 1994, mais déjà, c'était à contre-courant de ce qui se développait. C'est encore à contrecourant aujourd'hui de penser qu'une dépression - même si elle n'est pas pathologique, qu'il y ait des affects dépressifs ou de l'anxiété - ce puisse être une occasion de travail psychique, quelque chose sur lequel l'individu doit se pencher plutôt que...

G. D. S. :... que de l'évacuer.

Le DSM (Diagnostic Statistical Manual), s'en servir en clinique, c'est une tragédie. On peut le faire pour des statistiques, etc. La souffrance du patient, là-dedans, est de mieux en mieux décrite, mais de moins en moins recherchée dans son sens. De plus en plus, on met des mots, on met des diagnostics, mais on évacue la recherche du sens qu'il y a là-dedans. Tel symptôme va être évacué par telle pilule, plutôt que de rechercher le sens, ce qui est plus complexe, évidemment, mais qui est une nécessité si on veut travailler vraiment avec les patients, et chercher le sens unique que ça a pour tel individu à tel moment de sa vie.

Le psychiatre ne peut pas se permettre d'évacuer le subjectif ni l'objectif. Il doit se frayer un passage entre ces deux idéologies. Autrement dit, éviter le réductionnisme psychosocial, c'est-à-dire the brainless mind, mais également le réductionnisme biologisant, the mindless brain. Sinon, la psyché sera récupérée par la psychologie et le cerveau par la neurologie; donc, il n'y aura plus de place, d'une certaine manière, pour la psychiatrie.

R. L. : Mais est-ce que ce n'est pas ce qui est en train de se passer maintenant? Ça commence à ressembler à ça.

G. D. S. : Ça commence, justement...

Pourtant, la dépression est multifactorielle. Il y a une expérience traumatique, des facteurs génétiques, des traits de personnalité, et il peut y avoir ou ne pas y avoir quelque chose venant du support ou du manque de support de l'environnement. Ici encore une fois, je cite Henri Rey qui m’a beaucoup influencé, et que j'ai beaucoup aimé : «Aucune structure physique ne va expliquer, sans la structure psychologique, les fantasmes dépressifs. On peut changer la structure physique, mais ça ne changera pas les fantasmes dépressifs. »

R. L.: Je voulais aussi parler de votre texte sur la supervision collective parce que l'on retrouve dans ce texte votre intérêt pour les groupes.

G. D. S.: Oui, qui a commencé à Boston, déjà.

R. L.: Donc, on va poursuivre autour de votre intérêt pour les groupes, mais dans le cadre particulier que vous avez développé : celui de la supervision collective. Je pense 
que vous avez, pendant plusieurs années, fait des supervisions collectives pour l'enseignement de la psychothérapie psychanalytique. Et ça a donné lieu à la publication d'un texte dans le livre qui a été publié par les docteurs Wilfrid Reid et Pierre Doucet chez Gaétan Morin, en 1996: La psychothérapie psychanalytique. Dans ce livre, vous avez un texte où vous décrivez le dispositif que vous avez utilisé comme une enveloppe groupale et un contenant pour une rêverie à plusieurs.

G. D. S. : Ça, c'était dans le chapitre 38 de ce livre.

R. L. : Je voudrais peut-être juste mentionner, auparavant, qu'on utilise maintenant beaucoup le terme d'«intervision ». Je supervise des jeunes à l'université qui utilisent ce terme-là, c'est-à-dire que quand ils se rencontrent entre eux pour parler d'un cas, ils appellent cela intervision. Mais je pense que c'est vous qui avez introduit ce terme d'intervision. Dans la littérature, en tout cas, il me semble que vous êtes le premier chez qui on voit l'utilisation de ce terme, et du concept, peut-être, aussi.

G. D. S. : Oui durant des années où je voyais plusieurs résidents à Albert-Prévost, qui chacun présentait leur cas à tour de rôle, c'est devenu évident que chacun des participants et non seulement le responsable enseignant partageait leurs idées et fantasmes par rapport au cas présenté. Le terme supervision n'était plus adéquat et a été remplacé par celui plus approprié d'intervision. C'est venu à ce moment-là à Prévost puis par la suite ça a été adopté dans les séminaires, à l'Institut et à la Société, qui sont devenus de plus en plus quelque chose de semblable à ça.

Le biopsychosocial doit faire partie de la psychiatrie, parce que c'est la seule spécialité, en médecine, qui tient à ces trois aspects: le biologique, le psychique et le social. La personne ou la personnalité du traitant constitue l'agent thérapeutique. Puis, les participants du groupe d'intervision contribuent également par leurs propres associations libres, quand un membre du groupe présente son cas.

R. L.: C'est la rêverie à plusieurs.

G. D. S.: C'est ça, c'est la rêverie à plusieurs. C'était à l'époque, chez les résidents en psychiatrie, le seul endroit où ils pouvaient être écoutés quand ils présentaient leur cas et en même temps, écouter les autres du groupe, c'est-à-dire les autres résidents qui commençaient en psychiatrie. J'ai fait ça pendant 30 ans. J'ai commencé ça à Boston, mais aussi à Albert-Prévost, pendant une trentaine d'années. Ça s'appliquait non seulement aux résidants, mais aussi aux analystes en formation. Et puis, de plus en plus, aux analystes déjà formés; dans les séminaires qui ont cours aujourd'hui, je pense que c'est une formule que bon nombre ont adoptée. Chacun présente à tour de rôle un cas, et les participants associent librement à partir du cas. Le point central est de décoder l'accordage affectif dans le groupe et d'interpréter à partir de cela. Et ça, c'est vraiment tiré de Bion qui a donné beaucoup d'importance au climat affectif en tant que l'émotion suscitant la pensée. Mais il faut d'abord partir de l'émotion collective du groupe.

C'était, au fond, un processus de connaissance à plusieurs. Ce n'était ni une activité pédagogique comme telle, ni non plus une activité thérapeutique, mais je dirais, quelque chose qui est à la frontière des deux et qui exige une formulation spécifique. Un processus, donc, de connaissance, et à la fois un processus thérapeutique. 
R. L. : De connaissance?

G. D. S. : Oui, parce qu'on devient informé de ce qui se passe. C'est la connaissance, au fond, qui guérit dans l'analyse. C'est se connaître, finalement, qui est le processus thérapeutique; la psychanalyse étant, comme on vient de le dire, un processus de connaissance, mais en même temps un processus thérapeutique. La meilleure psychanalyse didactique, en fait, est la psychanalyse personnelle. C'est ce sur quoi l'on avait beaucoup insisté au congrès de Rome dont c'était justement le principal sujet.

L'intérêt de la supervision collective est d'atténuer le dilemme: est-ce qu'on est en train de traiter ou d'enseigner? Parce que dans la situation de groupe, il y a une interprétation du transfert et du contre-transfert, mais qui est faite tout autant par les co-supervisés que par celui qui est responsable du séminaire. Et c'est à partir de l'expérience émotionnelle du petit groupe qu'on essaie d'identifier ce qui se passe au cours du processus de connaissance. Il y a là une dilution du transfert, puisque c'est un transfert partagé; c'est non seulement un transfert sur le superviseur, mais aussi des transferts latéraux sur l'ensemble des membres du groupe. Il y a un transfert sur le groupe pouvant comporter des éléments primitifs maternels qui sont ainsi facilités.

La capacité de contenance en est le premier élément thérapeutique... Ce qui rejoint le processus psychanalytique de Meltzer, qui insiste beaucoup là-dessus : le premier outil thérapeutique, dans n'importe quel lien thérapeutique, c'est d'abord de recevoir, de contenir avant de transformer. Souvent avec des grands malades, par exemple les psychotiques, tout ce qu'on peut faire, finalement, c'est de «contenir».

Il est important qu'il y ait un libre choix du superviseur et des supervisés, ça ne peut pas être imposé. Il faut que les gens, dans les séminaires, aient une certaine affinité. Ça permet aussi d'observer différentes phases de l'évaluation du traitement des patients et les différents apprentissages des membres du groupe de supervision qui sont à différents moments de leur formation.

R. L. : Dans un même groupe...

G. D. S.: Oui.

J'ajouterais que ce que j'ai nommé l'intervision facilite la formation des supervisés pour devenir eux-mêmes superviseurs. Et on pourrait penser que la terminaison, c'est quand le supervisé a atteint lui-même une capacité d'autoanalyse. Il est prêt à ce moment-là, à partir lui-même, on his own. Le mode d'écoute se fait à partir de l' «enveloppe groupale» qui devient un contenant pour une rêverie à plusieurs. Chacun contribue à cette rêverie collective; ils vont dire: «Ah, ça me fait penser à... », ou bien, "J'ai rêvé l'autre jour de... » Et c'est souvent une pensée qui surgit de l'un et de l'autre, une contribution collective. C'est très différent des termes « supervision » et «contrôle» qui ont des connotations hiérarchiques : ces aspects-là sont mis de côté dans l'intervision, qui devient plutôt une consultation continue à plusieurs par rapport à ce qui est éprouvé par l'ensemble des participants du groupe.

C'est pourquoi j'ai proposé le terme intervision il y a plusieurs années; un terme qui a été repris par plusieurs, par après. Tout cela était inspiré de Bion, à partir de son livre Learning from Experience. On apprend à partir de l'expérience du terme latin 
expereri. Un homme expérimenté est quelqu'un qui a rencontré un péril et qui a surmonté le péril. Il devient ex-périmenté, littéralement.

Cet état de rêverie a été observé par Bion à partir de son observation de la mère et du nourrisson. C'est assez proche de l'attention flottante de Freud, et c'est une rêverie à plusieurs.

Je vous donne ici un exemple. Pendant neuf ans, à la Clairière, dans des groupes qui faisaient de l'observation, j'ai été inspiré par l'observation des garçons de 6 à 12 ans; j'ai aussi participé à un séminaire d'observation de nourrissons ce qui m'a beaucoup aidé par rapport à l'application de ces concepts-là. Je me souviens d'une situation. Il y avait une angoisse collective dans le petit groupe; il s'agissait d'un patient qui présentait un risque suicidaire. Trois participants sur 5 se sont mis, face à cette angoisse, à éprouver des borborygmes au cours de la supervision. Cependant parfois, il peut y avoir une nécessité d'une supervision individuelle, par exemple pour dénouer certaines impasses qui seraient plus personnelles.

L'intervision était peu répandue dans les instituts de psychanalyse autrefois, alors qu'aujourd'hui c'est fréquent dans les séminaires de la SPM. On s'est rendu compte que c'était utile: quelqu'un rapporte un cas et les autres associent spontanément, làdessus. Il y a de la supervision collective dans certains instituts en France également. R. L. : Je trouve ça intéressant parce que Bion dit, dans «Aux sources de l'expérience »: «L'expérience émotionnelle qui est celle de rapporter une séance d'analyse, n'est pas l'expérience émotionnelle de l'entrevue d'analyse, mais bien une nouvelle expérience émotionnelle, qui est celle de rapporter une séance d'analyse. » Et je trouve que ce que vous dites, c'est que c'est l'expérience émotionnelle vécue pendant cette supervisionlà qui est formatrice et qui va amener d'une part, à comprendre mieux la relation entre le patient et son thérapeute, mais va aussi être une formation pour tous les participants vivant l'expérience émotionnelle de la présentation par quelqu'un de son cas.

G. D. S. : Absolument.

R. L. : C'est une autre expérience émotionnelle. Et pour vous, c'est un filon de tous vos textes, l'expérience émotionnelle...

On peut peut-être passer à votre texte suivant qui va nous intéresser, celui que vous avez présenté à Turin, sur l'émergence de la pensée.

G. D. S. : C'est en 1998, oui.

R. L. : Pour vous, dans la continuation de Bion, l'émergence de la pensée est en lien direct avec l'expérience émotionnelle. Dans le processus vers la pensée, il y a l'expérience émotionnelle. Et vous développez ça dans ce texte que vous avez présenté à un congrès à Turin.

G. D. S.: C'était au Centennial en hommage à Bion organisé en 1987 par sa fille, Parthenope, parce que Bion est né en 1887. Le texte dont on parle a été publié en 1998 suite à sa présentation à Turin, où travaillait Parthénope, comme psychanalyste.

R. L.: Vous avez présenté à ce congrès-là, et le texte a été publié plus tard dans un livre sur Clifford Scott.

G. D. S. : C'est ça, c'était un des chapitres dans le livre sur Scott: Psychoanalysis and 
the Zest for Living. C'est Michel Grignon qui l'avait édité.

R. L. : Alors, on peut regarder les concepts que vous développez là parce que vous parlez de l'émergence de la pensée dont la source est l'expérience émotionnelle. Vous parlez de la digestion mentale... Il y a beaucoup de développements dans ce textelà. Quand vous avez présenté au congrès de l'Association des psychiatres du Québec, en 2009 au Manoir Richelieu, vous en avez repris un court résumé en français.

G. D.S. : Oui. Je vais en faire un résumé à partir du texte anglais qui a été publié dans ce livre: The Emergence of Thinking. Bion, as a link between Freud and the Neurosciences. Un lien entre Freud et les neurosciences. En fait, le vieux rêve de Freud d'unir le cerveau à l'esprit est repris, à l'occasion de ce congrès, dans ce texte où il est question de Bion en tant que lien entre Freud et les neurosciences. Dans un certain sens, il me semble que Bion réalise le rêve de Freud sur le lien entre la psychanalyse et les neurosciences. Les idées de Freud et de Bion sur l'émergence de la pensée se rejoignent. Le texte de 1911 de Freud, Les deux principes du développement mental, était déjà une amorce de cela. Pour Bion comme pour Freud, l' « expérience de satisfaction » est à la base de la transformation de l'animal humain en un humain pensant. L'identification projective, qui avait été déjà mentionnée comme étant un phénomène pathologique par Melanie Klein, Bion l'a transformée en un mécanisme normal pour la communication d'états de détresse du bébé ou d'un patient, dans un objet, mère ou analyste, un objet contenant et pensant pouvant être le transformateur de la détresse vécue sur un mode somatique.

La théorie de Bion est fondée sur la digestion mentale de l'expérience émotionnelle d'un bébé ou d'un patient, à l'intérieur d'un objet rêvant et pensant qui devient capable de faire la transformation et de contenir un état de détresse, ce que le bébé ou le patient était incapable de faire, c'est-à-dire passer d'un état de détresse physiologique vers un processus de pensée. Alors les idées de Bion rejoignent en quelque sorte certaines des idées des neurosciences et en particulier les idées d'Edelman, qui a eu le prix Nobel d'immunologie. La théorie d'Edelman affirme que le système immunitaire fonctionnerait par sélection. Les neurones les mieux adaptés à la situation seraient ceux qui seraient sollicités, qui se développeraient davantage et contribueraient au développement du cerveau. C'est intéressant, parce que là il y a un lien entre le développement de la psyché et le développement du cerveau. Il y a un parallélisme.

En effet pour Bion, le développement de la psyché va dépendre de la stimulation et de la digestion mentale des expériences émotionnelles appropriées, alors que les faits ou les expériences non digérées resteraient non transformées et demeureraient sous la forme de traces corporelles sensorielles non pensées. Pour Bion, l'apprentissage à partir d'une expérience émotionnelle vécue et pensée est celle qui contribue au développement du psychisme.

En résumé, la formulation de Bion au sujet de la pensée serait que la pensée commence par l'observation d'une expérience émotionnelle. Et cette expérience émotionnelle est d'abord éprouvée et ressentie dans le corps, dans le soma. Elle est appréhendée d'abord comme un événement corporel qui sera transformé en une expérience émotionnelle par le biais de la fonction alpha. Sinon, ces expériences 
seraient évacuées sans transformation dans le corps. Ceci, essentiellement, est la théorie psychosomatique de Bion.

R. L.: Je pourrais tout de suite vous interrompre... Ceux qui on travaillé avec vous vous ont souvent entendu dire comment une pensée vraie s'appuie sur les trois sens du mot sens. C'est ce que vous venez de décrire. Peut-être que vous pouvez rappeler quels sont ces trois sens du mot sens.

G. D. S.: C'est d'abord la sensation, le corps. Puis le sentiment, l'émotion.

R. L.: L'expérience émotionnelle.

G. D. S. : Voilà. Et finalement, le sens du mot. Les trois sens du mot sens : c'est vraiment Bion qui en a fait la théorie.

R. L.: Et donc, cela a une implication directe pour l'analyste...

G. D. S.: Et pour une pensée vraie. Une pensée vraie doit réunir ces trois sens. Parce qu'on sait qu'il y a des patients qui parlent, qui parlent, ou qui sont plein d'émotions, mais il n'y a pas de sens qui est donné à cela. Ou bien ils sont en train de vivre des sensations corporelles, tout à coup en séance, il peut y avoir un malaise et ce n'est pas incorporé. C'est le cas de le dire, «incorporé». C'est dans le corps, mais ce n'est pas incorporé dans le travail qui est fait en séance, alors que ça devrait l'être: pourquoi, à ce moment-là, il y a telle sensation qui apparait?

R. L.: Donc, il y a toute une manière d'écouter, une écoute de l'analyste, qui se déplace. C'est-à-dire qui n'est pas que sur le langage et sur le sens, mais qui se déplace sur l'observation de l'éprouvé émotionnel et même de l'éprouvé corporel.

G. D. S. : Absolument. Une caricature de cela est: « des mots, des mots, des mots, que des mots. » Il y a une chanson aussi : «Paroles, paroles, paroles »... En voulant dire: c'est des beaux mots, mais c'est juste une évacuation de paroles, sans qu'il y ait un soubassement qui corresponde à une pensée vraie qui, elle, doit contenir les trois sens du mot sens: sensation, sentiment et sens des paroles exprimées.

Plusieurs observations en pratique clinique sont discutées dans le texte en question, de même que plusieurs observations en biologie, en embryologie et en neurosciences sont présentées. Ces diverses observations viennent soutenir la conviction que le cerveau et la psyché, et en particulier le système nerveux et le système alimentaire, ont été étroitement liés tout au cours de l'évolution. Edelman, en ce sens, pourrait avoir réussi le projet de Freud de réunir la science psychologique aux sources des sciences de la nature.

R. L.: Donc, l'appareil à penser comme analogie avec l'appareil digestif, ce n'est pas uniquement une métaphore. Ça va plus loin que ça dans ce que vous dites. L'appareil digestif n'est pas seulement utilisé comme métaphore de la pensée. Vous dites, d'après Edelman, qu'il y a même une source commune dans le développement, entre le développement de l'appareil digestif et le cerveau.

G. D. S. : J'ai mis en appendice un certain nombre de références à des textes dans lesquels cela est développé.

R.L.: On devrait aussi parler d'un autre texte, celui sur l'identification projective. On pourrait en parler maintenant puisque dans le texte sur l'émergence de la pensée, vous mentionnez comment Bion a apporté un élargissement du concept d'identification 
projective. Il ne s'agit pas seulement d'évacuer de façon violente des aspects qui ne peuvent pas être contenus, mais c'est toute la question de la modalité primitive de communication: dans la communication normale, il y a identification projective.

G. D. S. : C'est ça, le volume 11, nº.1, à l'automne 1998.

R. L. : Vous avez écrit dans le Bulletin de la Société psychanalytique de Montréal un texte sur l'identification projective. Est-ce que je me souviens correctement que c'était une réponse ou une réaction à une présentation faite à la Société?

G. D. S.: Oui, suite à ça. Parce que ce thème-là a été souvent abordé, mais il y a une confusion conceptuelle remarquable. Moi, je me suis inspiré beaucoup de Meltzer à ce sujet-là. Et j'ai essayé, justement, de distinguer le pourquoi de l'identification et ce qu'il en est. La question avait été posée quand Meltzer est venu à Montréal. On m’avait demandé de présenter un cas parce qu'on savait du côté anglais mon intérêt pour les textes de Meltzer.

R. L. : Meltzer était venu au Quebec English?

G. D. S. : Oui, j'allais souvent du côté de Quebec English et on était au courant, on m'avait demandé de présenter un cas clinique. Je ne veux pas en dire trop sur ce cas clinique assez particulier.

On avait posé la question à Meltzer: «Y a-t-il moyen de nous dire de façon simple, à des gens qui ne connaissent pas grand-chose là-dessus, c'est quoi l'identification projective? » Et Meltzer avait répondu en disant qu'il n'y a pas de façon simple de parler de ce concept-là. Parce que d'abord, il a été introduit par Melanie Klein en 1946, et à ce moment-là, c'était perçu par elle comme un mécanisme psychotique; il s'agit alors essentiellement de la projection d'une part de soi qu'on ne peut pas reconnaître en soi, et qu'on adresse à un autre. Ça, c'est un phénomène ordinaire où l'on accuse l'autre. Le sujet fait une projection d'une part de soi dans l'autre et se comporte ensuite par rapport à l'objet dans lequel il a mis une part de lui-même comme si l'autre, l'objet, était devenu à l'image de la projection mise en lui par le sujet; mais c'est lui, le sujet qui a mis une partie de lui dans l'autre.

R. L.: Ça, donc, ce serait la part identificatoire. Il identifie l'autre...

G. D. S. : Il identifie l'autre à partir de ce qu'il a projeté de lui à l'autre.

R. L.: C'est important parce qu'il y a l'aspect projectif et il y a l'aspect identificatoire.

G. D. S.: Oui. Alors, le sujet fait une projection d'une partie de soi sur l'autre. Et ensuite, il se comporte, vis-à-vis l'objet, à la manière colorée par ce qu'il a projeté dans l'autre.

Freud et Ferenczi avaient déjà parlé de l'identification introjective. Dans l'identification introjective, le sujet est enrichi par ce qu'il a pris de l'autre pour le mettre en soi. C'est ainsi qu'il y a du développement, d'ailleurs. Le sujet est enrichi par ce qu'il a pris de l'objet.

Dans l'identification projective, le sujet est appauvri d'une certaine manière parce qu'il a mis une part de lui, une part qu'il ne veut pas reconnaître en lui, dans l'autre. Alors, identification projective ou identification narcissique, il y a un certain lien. Par exemple, dans l'état amoureux, souvent, c'est aussi une partie de soi qu'on va imaginer être dans l'autre. Mais dans ce cas-là, on le colore avec une part de bon 
qu'on a soi-même et qu'on attribue à l'autre. Le texte discute justement des différentes variations qu'il y a dans les relations entre un sujet et un objet à partir de ce processus-là.

R. L.: De l'identification projective.

G. D. S.: Oui, de l'identification introjective et projective. Il est très important de faire une clarification de ces deux concepts.

R. L.: On va maintenant aller à un autre texte, mais on reviendra sur Meltzer. Il faudra qu'on en parle un peu, parce qu'il a été très important pour vous.

G. D. S.: Ah oui, son œuvre...

R. L.: Oui, on va y revenir... Peut-être qu'on pourrait parler du texte sur la confidentialité. Ce texte-là, vous l'avez publié en 2003. C'est un chapitre d'un livre qui est paru chez Analytic Press et qui a été édité par Charles Levin, Allanah Furlong et Mary Kay. Ça a été important pour vous, parce que d'abord, ce texte-là vous a valu un prix, le prix Miguel Prados, en 2003, de la Société canadienne.

C'est un texte dans lequel vous parlez de deux cas cliniques. Vous développez la nécessité de protéger la situation analytique des empiètements extérieurs. Et je me suis demandé pourquoi vous vous étiez intéressé à cela. On le comprend un peu à travers vos textes cliniques, mais qu'est-ce qui vous avait amené à réfléchir à cette question-là?

G. D. S. : C'est à cause d'une patiente que j'ai appelée Pandora. C'est une patiente qui avait été laissée de façon abrupte par un analyste qui lui avait dit de continuer avec un analyste plus expérimenté.

Et puis, la patiente avait dénoncé son analyste à son association professionnelle. Et lui, tout ce qu'il lui avait dit c'est: "Voyez un autre analyste.» La patiente avait décidé de poursuivre son ex-analyste au civil.

En fait, le père de cette patiente-là était psychotique et la mère avait face à sa fille, la patiente, adopté une attitude : «Ignore-le. Ignore ça. On ne parle pas de ça.»

Un analyste précédent l'avait laissée parce qu'il n'a pas voulu s'embarquer làdedans. Et puis, elle a été furieuse, évidemment.

En tous cas, son besoin de dénoncer était lié, au fond, à son désir de reconnaître la psychose du père, et au fait que la mère avait suggéré d'ignorer ça. Une tentative de dénoncer liée au failing object, le père et l'analyste précédent, mais aussi, probablement, la mère qui voulait cacher tout ça. Alors, la patiente voulait poursuivre son premier analyste.

Une tentative de la patiente, de m'amener dans une bataille externe, en dehors de ses conflits internes. Enfant, elle avait dû subir les injonctions maternelles: «Occupe-toi pas du père. Occupe-toi pas de ça.» Alors, mon dilemme c'était: comment arriver à préserver la confidentialité d'une part, alors qu'elle voulait que je témoigne contre l'autre analyste qui l'avait laissée.

R. L.: Elle voulait vous amener à témoigner dans sa poursuite.

G. D. S. : Dans sa poursuite. Et donc, d'une certaine façon, j'étais coincé à plusieurs égards. Parce que garder la confidentialité de ce qui se passait entre nous, ne pas sortir du cadre, donc, c'était de son point de vue, l'abandonner. Failing her in her external 
life, d'un côté. J'étais coincé parce que là, si je refusais de témoigner à la Cour, elle pouvait me poursuivre, $\mathrm{j}$ 'aurais pu être soumis à un contempt of court.

R. L.: Oui, c'est un mépris de cour.

G. D. S. : Un mépris de cour. Mais en même temps, témoigner allait à l'encontre du travail que je voulais faire avec elle. Alors, j'étais coincé de tous côtés, si on veut. Et également, sortir du cadre, c'était pour moi du self contempt en tant qu'analyste. Donc je me sentais coincé de partout.

À cause de cela, I was prepared to risk going to jail. J'étais prêt à cela. Quand j'ai dit que j'étais prêt à aller en prison si j'étais condamné, mais que je ne témoignerais pas et que je dirais au juge pourquoi, c'est là qu'elle a changé. Elle a demandé à son avocat de ne plus m'envoyer le subpoena. J'étais soulagé et l'analyse a pu se poursuivre. I was in a confusion like the patient.

Mais la psychanalyse est un processus de transformation. À preuve, par exemple, le mot «transformation». Je me souviens d'avoir discuté de cela avec Henri Rey à l'époque. Dans toute l'œuvre de Freud, l'un des mots qui apparaît le plus souvent, qui apparaît 527 fois, c'est le mot «transformation». Donc, essentiellement, la psychanalyse c'est un processus de transformation.

Une fois que ça a été réglé, l'analyse a pu continuer. Enfin, on peut parler de sa résistance à éviter ses conflits internes. Une fois que tout ça a été résolu, plus tard, elle s'est rendu compte qu'en fait, no one is perfect. Et elle a commencé à éprouver, enfin, une certaine compassion pour ses parents, pour ses analystes antérieurs aussi, et également pour moi.

À partir de ce moment-là, la situation analytique a pu évoluer. Then, a more trusting relationship could now be established. On a pu vraiment être en analyse et s'occuper enfin de ses conflits internes où une certaine compassion a pu apparaître pour ses parents et pour moi, ainsi qu'une diminution du persécuteur interne qui exigeait d'elle la perfection.

Cet exemple parle de l'importance de situer la situation analytique, qui est à la fois à l'intérieur et à l'extérieur de la loi. La confidentialité est un privilège... En latin la loi privée, c'est privus. Privus le, c'est une loi privée. Dans la loi romaine, l'avocat peut refuser de témoigner contre son client; tout comme un esclave, à l'époque, ne pouvait témoigner contre son maître. Donc, il y a un certain privilège qui remonte en fait à la loi romaine. J'ai consulté des avocats, moi, à cette époque-là. Je voulais continuer l'analyse, mais je ne voulais pas me faire coincer.

Charles Levin m'a aidé aussi. Notre code civil remonte au fond à la loi romaine. Je ne savais pas ça. L'analyste est dans un état de servitude et ne doit pas témoigner. La psychothérapie et la psychanalyse sont différentes de la psychiatrie. Dans la psychiatrie, les gens vont témoigner C'est une adaptation à la société. Mais la psychanalyse a à faire avec le monde interne et non pas le monde externe. La loi doit s'occuper des actions externes et non pas du monde interne des patients.

R. L.: C'est là que vous faites la différence, quand vous dites que c'est à la fois dans la loi... et en dehors.

G. D.S. : C'est ça. La loi régule les oppositions entre les groupes d'individus alors que 
la psychanalyse doit intégrer les aspects conflictuels à l'intérieur de la même personne, c'est-à-dire du patient. C'est une différence fondamentale.

La psychanalyse est un processus de développement transformationnel. Ce qui est amené en psychanalyse, ce sont les parties non intégrées de la personnalité. Citant Bion : "C'est un appareil pour penser les pensées. Penser, c'est une évolution à partir des sensations corporelles vers des pensées abstraites. » Ça, c'est la grille de Bion. Dans sa grille, justement, il y a des choses concrètes et on va vers de plus en plus d'abstraction; y compris dans les mathématiques.

Et il y a cette phrase qui m'est venue, et que j'entends encore Meltzer dire, après une question qui lui avait été posée quand il est venu à Montréal invité par Quebec English: "Thinking commences with the observation of an emotional experience, and this emotional experience is right there in the body, the alph a function works right there in the body. » Il y aurait donc un parallèle à faire, entre la digestion de nourriture et la digestion des pensées. L'appareil à penser se serait calqué sur l'appareil digestif. C'est pourquoi les borborygmes en séance psychanalytique peuvent signaler un sens. La situation analytique rejoint ainsi le Socrate du «connais-toi toi-même ». Le dilemme constant, pour survivre, serait à la fois de se connaitre, mais en même temps, avec la terreur de se connaître. On est des humains coincés là-dedans.

En psychanalyse, le rêve est ce que nous avons de plus proche de notre vérité. C'est la pierre d'assise de la recherche de notre vérité. Le patient et l'analyste doivent se maintenir dans deux états d'esprit: le patient en association libre et l'analyste en état de rêverie. In our body and in our mind. L'analyste doit rêver ce que le patient est incapable de rêver. Et pour cela, il doit y avoir une certaine conscience altérée en cours de séance. On doit être capable de se mettre dans un certain état où on est libre de recueillir ou d'accueillir les émotions réciproques. Pour Bion, le bébé et le patient évacuent respectivement dans la mère et dans l'analyste ce qu'ils ne peuvent pas penser. Et la capacité négative dont il parle, c'est cette habileté à rester dans l'incertitude sans chercher de raison. L'analyste doit attendre, recevoir les projections du patient et doit rêver ce que le patient ne peut pas rêver. C'est cela la fonction alpha de Bion. Et Bion cite Keats (décédé en 1917):

«Beauté et vérité,

vérité et beauté,

c'est tout ce que vous devez connaître,

c'est tout ce qu'il y a à connaître.»

Meltzer mentionne que dans les rêves, souvent, on peut observer une progression. Les projections peuvent être faites dans des machines inanimées, ensuite dans des animaux, ensuite dans des membres de la famille, et ensuite, à l'intérieur de soi par intégration des aspects de sa personnalité. Ce qui rejoint ce que Freud disait: «chaque partie du rêve est une partie de la personnalité du rêveur ». Les rêves sont des projections, et moins on veut se connaitre ou savoir, plus on va projeter, et de plus en plus loin. On peut voir au cours de l'analyse que les projections deviennent plus proches, plus faciles à repérer.

Un des buts de la psychanalyse serait de reconnaître qu'on est l'auteur de ses 
rêves. Pour cela, il y a nécessité d'un espace privé (free space of mind), sans action et sans jugement, pour qu'il puisse y avoir ce travail de transformation. Bion recommandait d'être totalement dans le présent, sans mémoire et sans désir, sans passé et sans futur. Et Freud proposait de ne pas prendre de notes durant les séances.

R. L.: On est passé à travers tous les textes les plus importants, mais j'aimerais qu'on parle de Bion. Parce que dans vos écrits, Bion est, avec Freud, l'auteur le plus cité, le plus travaillé. Mais aussi Henri Rey. On a mentionné comment il a été très important; on a sa photo ici dans votre bureau. J'aimerais aussi que vous parliez un peu de Meltzer, parce que ce sont des gens dont vous avez travaillé les textes, mais vous avez eu une relation personnelle avec les deux.

G. D. S. : Meltzer je suis allé à une célébration de ses écrits à Tavistock quelque temps après son décès, il y avait eu une commémoration. J'avais eu aussi une période d'analyse avec lui.

R. L. : Donc, vous l'avez connu mais comment vous avez pu organiser de faire une tranche d'analyse avec lui?

G. D. S.: Je suis allé quelque temps à Oxford où il habitait. Madame Bion habitait aussi à Oxford. Je l'avais rencontrée quelquefois parce qu'elle était venue avec sa fille Parthénope quand Parthénope avait organisé le Centennial, en l'honneur de son père, à Turin où elle habitait. J'étais allé et j'avais fait la présentation dont on a parlé plus tôt. J'ai plusieurs photos de madame Bion, mais aussi de son fils et sa fille Parthénope. R. L. : Madame Bion, c'est Francesca, c'est ça?

G. D. S. : C'est ça, Francesca, qui était plus jeune que lui. On a gardé une correspondance dans le temps des fêtes. Quand j'étais allé consulter Meltzer, je l'ai aussi visité; elle aussi vit à Oxford. Bion se proposait de vivre à Oxford, mais il est décédé juste avant. Bion et Meltzer, devaient faire un groupe là-bas ensemble, mais il est décédé juste avant.

Bion devait aussi retourner aux Indes; il n'était jamais retourné aux Indes où il est né. Il avait quitté les Indes à 8 ans, on l'avait envoyé par bateau. Sa mère l'avait accompagné jusqu'à Londres, mais les enfants des colons anglais allaient faire leurs études en Angleterre. Son père était ingénieur dans les locomotives. Les gens voyagent par trains là-bas, c'est les Anglais qui ont installé cela ; et le père de Bion était un de ceux-là. Ils sont restés là-bas longtemps, mais lui (Bion), à 8 ans, ils l'ont envoyé dans les bonnes écoles d'Angleterre. Il n'était jamais retourné mais là, il se proposait d'aller à Bombay. C'était en novembre, et il devait y aller en janvier. Il est décédé deux mois avant!

R. L.: Donc, il n'est jamais retourné aux Indes.

G. D. S. : Il n'est jamais retourné dans le pays de son enfance où il s'apprêtait à aller, alors qu'il avait voyagé considérablement. Il n'était jamais retourné aux Indes, c'est quand même remarquable.

R. L. : Pourquoi il n'est pas allé...

G. D. S. : Et pourquoi il est mort à ce moment-là, alors qu'il était en bonne santé. Il est décédé à 82 ans, juste au moment où il se préparait à retourner dans le pays dont il avait été « expulsé » à 8 ans. C'est quand même triste... On pourrait poser toutes 
sortes de questions... Parce qu'il était en forme.

R. L.: Oui, d'ailleurs, il n'est pas mort d'une longue maladie, il a eu quelque chose d'assez subit...

G. D. S.: Oui, une leucémie.

C'est une maladie sanguine brutale qui l'a emporté en novembre, alors qu'il partait en janvier.

R. L.: En fait, il était revenu d'abord des États-Unis pour s'installer...

G. D. S. : Il était allé aux États-Unis plusieurs fois. Il a vécu en Californie un bon bout de temps, la Californie qui lui rappelait, à bien des égards, l'Inde.

R. L.: Et là, il venait de revenir s'installer en Angleterre et...

G.D. S.: En fait, il avait pris une petite distance de Londres, pour s'installer à Oxford. C'est à peu près à 30, 35 kilomètres. Et ça voyage bien, c'est très rapide, c'est à la périphérie de Londres.

R. L.: Alors, vous, quand vous avez fait des séances avec Meltzer, c'était chez lui; il avait son cabinet à la maison.

G. D. S.: C'était chez lui, oui, à Oxford. J'ai failli rester pour plus longtemps, mais finalement, non. J'ai passé quelque temps mais des séances très fécondes.

R. L.: Oui, le temps de faire l'expérience d'une analyse avec lui.

G. D. S. : Ah oui. J'ai pris beaucoup de notes. Comme j'ai déjà mentionné j’avais présenté un cas devant lui quand Quebec English l'avait invité quelques années auparavant. Comme il était dans la même filière que Bion, j'avais étudié ses écrits dans un séminaire que j'avais formé et fais des présentations à ce sujet.

R. L.: Et comment c'était de présenter un cas à Meltzer?

G.D.S.: Sa formule, c'était toujours la même. Disons, un résumé d'une page de l'histoire et puis une séance. That's it. Et à partir de ça, il enchaînait là-dessus. C'était une séance ou peut-être une série d'une semaine... Moi, ça m’avait bien impressionné et touché. Il y avait plusieurs personnes qui ont été influencées par lui, d'un peu partout. Y compris Ferro.

R. L.: C'est intéressant parce que vous dites comment Meltzer a inspiré probablement Antonino Ferro, qui est un analyste d'enfant, probablement un de ceux qui a la plus grande notoriété actuellement comme analyste d'enfants. Meltzer lui-même s'est beaucoup intéressé à l'analyse d'enfants. Je ne crois pas qu'il en faisait lui-même, mais il donnait des supervisions, des séminaires...

G. D. S. : Il a donné des supervisions un peu partout. En Europe, il se déplaçait. Il est allé souvent en Italie où il était très connu. Dans le nord de l'Italie, surtout: Turin, mais aussi Milan, une grande ville, près de l'Adriatique...

R. L.: Donc, par exemple, dans son livre Explorations dans le monde de l'autisme, des cas sont analysés ou enfin développés par Meltzer, mais à partir de présentations de collègues, qui eux voyaient des enfants. Mais ça me ramène à vous...

G. D. S. : J'ai participé à un séminaire d'observation de bébé. J'allais au séminaire pendant à peu près huit ans, à Albert-Prévost, du côté des enfants...

R. L.: Le séminaire de Johanne Giasson.

Vous-mêmes, vous n'êtes pas analyste d'enfants, mais vous vous êtes beaucoup inté- 
ressé à l'analyse d'enfant.

G. D. S. : Oui. Il y a, une fois par mois à la Société, un groupe de psychanalyse d'enfants qui présente des cas.

R. L.: Du côté anglais.

G. D. S. : Oui, Michel Grignon est là. J’y vais régulièrement. Je trouve ça tout à fait intéressant.

R. L.: Vous avez toujours eu cet intérêt, mais en tant que didacticien de l'Institut vous êtes allé plus loin, parce que vous avez écrit une lettre pour défendre le fait qu'on puisse considérer un cas d'enfant comme un cas contrôle, pour un candidat qui fait sa formation à l'Institut.

G. D. S. : Oui tout à fait.

R. L. : Ça date de quelques années, mais c'est vous qui avez défendu cette idée-là comme si pour vous, l'expérience avec les enfants était fondamentale pour un analyste d'adultes. Et pour vous, une analyse d'enfant, ça vaut une analyse d'adulte.

G. D.S. : J'ai été neuf ans à la Clairière, et je trouve cela dramatique que ça ait fermé. Parce que moi, pendant neuf ans, j’y allais une journée par semaine; c'était ce qu'ils appelaient un internat pour garçons de 6 à 12 ans.

R. L. : J'ai été là aussi à la Clairière, à peu près en même temps que vous...

G. D.S. : J'étais dans le groupe des Cosmos. J'ai trouvé ça une tragédie qu'on ait fermé cette institution. Moi, $j$ 'ai vu des miracles au cours des neuf années. Je voyais les nouveaux arrivants et je discutais avec les membres du personnel. Michel Lemay était là aussi. Sa femme également.

R. L. : Écoutez, je pense qu'on a fait le tour. Je vous remercie de tout ce temps...

G. D.S.: Je vous remercie de m'inscrire dans votre série d'entretiens, je trouve ça tout à fait important et intéressant.

R. L.: C'est important que l'on vous ait dans cette série d'entretiens. Moi, j'ai relu vos textes mais vous les avez relus aussi. Vous nous avez donné beaucoup de temps. Merci.

Réal Laperrière

1209 , Fleury Est, app. A

Montréal, Québec, H2C 1R2

\section{Textes de Guy Da Silva consultés pour l'entretien}

1963, The role of the father with chronic schizophrenic patients, Canadian Psychiatric Association Journal, $8,3,190-203$.

1965, Loveliness and death of an 81-year-old man. A revealing case history of depression in old age, Geriatric Focus, 4, 7, 2-6.

1965, Discussion: psychotherapy of aged patients, The loveliness and death of an 81-year-old man, Geriatric Focus, 4, 8, 2-6.

1966, Considérations sur le vieillissement et la psychothérapie des personnes âgées, Laval médical, 37, 199207.

1967, Considérations psychodynamiques sur le vieillissement, L’Union médicale du Canada, 96, 570-577.

1967, The loveliness and death of an old man : three years psychotherapy of an eighty-one-year-old depressed patient, Journal of Geriatric Psychiatry, 1, 1, 5-27.

1969, Essai d'une définition de la fonction paternelle, Interprétation, 3,1 et 2, 264-275. 
1973, L'analyse en tant que consolidation de la phase de séparation-individuation, Société psychanalytique de Montréal, (non publié).

1974, La situation d'être malade. Essai d'une théorie de la psychothérapie, L'Union médicale du Canada, 103, 1920-1926.

1990, Borborygmi as markers of psychic work during the analytic session. A contribution to Freud's «experience of satisfaction » and to Bion's idea about the digestive model for the thinking apparatus, The International Journal of Psychoanalysis, 71, 4, 641-659.

1994, Deuil et dépression mélancolique: travail psychique et réflexions sur la médication, Pavillon AlbertPrévost (non publié).

1996, La supervision collective dans l'enseignement de la psychothérapie psychanalytique: enveloppe groupale pour une rêverie à plusieurs, in Doucet, P., Reid, W., éd., La psychothérapie psychanalytique. Une diversité de champs cliniques, Gaëtan Morin, Montréal, 612-627.

1998, L’identification projective, pour quoi faire?, Bulletin de la Société psychanalytique de Montréal, 11, 1, 7-12.

1998, The emergence of thinking: Bion as the link between Freud and the neuro-sciences, in Grignon, M., éd., Psychoanalysis and the Zest for Living. Reflections and Psychoanalytic Writings in Memory of W.C. Scott, Binghamton, ESF Publishers, 189-202.

2000, Les chercheurs d'or, Bulletin de la Société psychanalytique de Montréal, 12, 3, 13-17.

2003, Confidentiality in psychoanalysis: a private space for creative thinking and the work of transformation, in Levin, C., Furlong, A., O’Neil, M.K., eds., Confidentiality: Ethical Perspectives and Clinical Dilemmas, Hillsdale, The analytic press inc., Publishers, 151-165.

2007, Discussion du texte de Christian Godbout: "Survivance et capacité négative ». La question de l'endurance en psychanalyse, Bulletin de la Société psychanalytique de Montréal, 19, 1, 18-20.

2007, Les bienveillantes de Jonathan Littell. Notes de lecture, Bulletin de la Société psychanalytique de Montréal, 19, 3, 49-50. 